\title{
Analyses of the LMC Novae
}

Karen M. Vanlandingham, Greg J. Schwarz, Sumner Starrfield

Department of Physics \& Astronomy, Arizona State University, Tempe, AZ 85287-1504, USA

Peter H. Hauschildt

Department of Physics and Astronomy, University of Georgia, Athens, GA 30602-2451, USA

Steven N. Shore

Department of Physics and Astronomy, Indiana University South Bend, 1700 Mishawaka Ave, South Bend, IN 46634-7111, USA

George Sonneborn

Laboratory for Astronomy and Solar Physics, Code 681, NASA/GSFC, Greenbelt, MD 20771, USA

\begin{abstract}
.
In the past 10 years, 6 classical novae have been observed in the Large Magellanic Cloud (LMC). We have begun a study of these objects using ultraviolet spectra obtained by IUE and optical spectra from nova surveys. We are using the results of this study to further our understanding of novae and stellar evolution.

Our study includes analysis of both the early, optically thick spectra using model atmospheres (Hauschildt et al. 1992), and the later nebular spectra using optimization of photoionization codes (Ferland 1996; James \& Roos 1993). By analysing all the LMC novae in a consistent manner, we can compare their individual results and use their combined properties to calibrate Galactic novae. In addition, our studies can be used to determine the elemental abundances of the nova ejecta, the amount of mass ejected, and the contribution of novae to the ISM abundances. To date we have analysed Nova LMC 1988\#1 (Schwarz et al. 1998) and Nova LMC 1990\#1 (Vanlandingham et al. 1999), and have obtained preliminary results for Nova LMC 1991. The results of this work are presented in this poster.
\end{abstract}

\section{Introduction}

The metal content of the LMC is known to be sub-solar and varies as a function of location within the cloud. A detailed abundance analysis of the ejecta of the LMC novae provides important information concerning the effect of initial 
metal abundances on energetics of the nova outburst. Since the distance to the LMC is well known, many important parameters of the outburst, such as the luminosity, can be absolutely determined. In addition, both galactic and extragalactic novae have been proposed as potential standard candles. Recent work by Della Valle \& Livio (1995) has improved on the standard relations by including novae from the LMC and M31. Unfortunately, the dependence of the nova outburst on metallicity has not been well-studied. Recent theoretical work by Starrfield et al. (1998) indicates that the luminosity of the outburst increases with decreasing metal abundances. If there is a dependence of luminosity on metallicity, it will have to be determined before novae can be used as standard candles.

\section{Results}

Our nebular analysis results for LMC $1990 \# 1$ show that all elements which are represented in our emission line spectrum are enhanced relative to solar material. We find a high N/O ratio for LMC 1990\#1, which is indicative of an outburst on a high mass white dwarf (Starrfield et al. 1992). Our estimate of the ejected mass is $\mathrm{M}_{e j} \simeq 4 \times 10^{-4} \mathrm{M}_{\odot}$.

We have determined the high Z metal abundances (e.g., iron) of LMC 1988 \#1 and LMC 1991 from model atmosphere analyses of optical and UV spectra obtained near visual maximum. We find an iron abundance of $1 / 3$ solar in LMC $1988 \# 1$, typical of the $\mathrm{LMC}$, but $\mathrm{Fe}=0.1 \mathrm{Fe}_{\odot}$ in $\mathrm{LMC} 1991$. This is consistent with the locations of these novae within the LMC. The visual lightcurves derived from the best fit synthetic spectra are in excellent agreement with the observed lightcurves when both novae were optically thick. In addition, the bolometric lightcurves show that LMC 1988\#1 and LMC 1991 were significantly brighter than the Eddington luminosity for a $1.4 \mathrm{M}_{\odot}$ white dwarf.

\section{References}

Della Valle, M., \& Livio, M. 1995, ApJ, 452, 704

Ferland, G.J. 1996, Department of Physics \& Astronomy Internal Report (University of Kentucky)

Hauschildt, P.H., Wehrese, R., Starrfield, S., \& Shaviv, G. 1992, ApJ, 393, 307 James, F., \& Roos M. 1993, MINUIT, CERN Program Library D506

Livio, M. 1992, ApJ, 393, 516

Schwarz, G.J., Hauschildt, P.H., Starrfield, S., \& Sonneborn, G. 1998, MNRAS, 300,931

Starrfield, S., Shore, S.N., Sparks, W.M., Sonneborn, G., Truran, J.W., \& Politano, M. 1992, ApJ, 391, L71

Starrfield, S., Truran, J.W., Wiescher, M.C., \& Sparks, W.M. 1998, MNRAS, 296,502

Vanlandingham, K.M., Starrfield, S., Shore, S.N., \& Sonneborn, G. 1999, MNRAS, submitted 